\title{
Passivity-based Voltage and Frequency Stabilization in AC microgrids*
}

\author{
Pulkit Nahata and Giancarlo Ferrari-Trecate ${ }^{1}$
}

\begin{abstract}
In this paper, we investigate the stability of an islanded AC microgrid (ImG) composed of Distributed generation units (DGUs), dynamic power lines, and loads. Each DGU is equipped with a local controller to ensure desired voltage and frequency in the ImG. Different from the previous works [1], we account for the dynamics of $R L$ power lines and consider generic interconnections of loads and DGUs. The stability of the ImG hinges on the passivity of all components with specific input-output pairs and the properties of the electrical interconnections. For the DGUs, we provide necessary and sufficient conditions for the existence of control gains guaranteeing passivity. In addition, we show that controllers decoupling the direct- and quadrature axis components of the electric variables can never render the DGU passive. Theoretical results are backed by simulation studies.
\end{abstract}

\section{INTRODUCTION}

Islanded microgrids are spatially-distributed electric systems composed of multiple small subsystems, for example, flexible loads, distributed generation units (DGUs), and storage units, interconnected to each other through an electrical network. A central theme in islanded AC microgrids (ImGs) is to ensure overall voltage and frequency stability through decentralized control of each DGU [2]. Droop-based voltage stabilization is a commonly used decentralized approach but is plagued by load-dependent voltage deviation, propagation of voltage error along resistive transmission lines, and presence of steady state voltage drifts [2], [3], [4], [5], [6]. Droop-free controllers have also been proposed. However, the voltage and frequency stability is often shown using simplifying assumptions on ImG topology, DGU models, and line dynamics [7], [1], [8].

This work focuses on the droop-free control proposed in [1], where a decentralized control architecture is described Local controllers are synthesized by solving a convex optimization problem requiring solely the local parameters of the corresponding DGU. However, the stability of the ImG has been shown under the following assumptions: (i) Quasi-Stationary-Line (QSL) approximation [9], where line inductances are neglected (ii) loads are attached only to the terminals of the DGUs, and (iii) loads are modeled as ideal current generators.

In this paper, we show that the controllers proposed in [1] can also stabilize an ImG with i) dynamical model of RL lines with arbitrarily large inductances, and (ii) constant impedance and constant current loads (commonly referred

\footnotetext{
*This work has received support from the Swiss National Science Foundation under the COFLEX project (grant number 200021_169906).

${ }^{1}$ Pulkit Nahata, and Giancarlo Ferrari-Trecate are with the Automatic Control Laboratory, École Polytechnique Fédérale de Lausanne (EPFL), Switzerland. Email addresses: \{pulkit.nahata, giancarlo.ferraritrecate\} eepfl.ch
}

as ZI loads) appearing at generic positions within the ImG. To this aim, we use passivity theory, which provides a compositional framework for the analysis of large-scale systems [10], [11], [12], [13]. In particular, we extend the stability argument proposed in [14], [15] for DC microgrids and show that the overall stability of the ImG hinges solely on the passivity of its subsystems, that is, DGUs, loads, and power lines. It must be noted that stability analysis in the $\mathrm{AC}$ case is not a straight-forward extension of the DC case as one must handle balanced three-phase signals or, equivalently, their $d q$ representation [16]. In addition, these $d q$ components are inherently coupled, making the analysis more complex. Different from [14], [15], we provide sufficient conditions for the passivity of the DGUs and show that they can be passivated if and only if the storage functions conform to a particular structure. Moreover, we show that decoupled controllers handling the $d$ and $q$ components separately cannot passivate a DGU. Finally, we show the overall asymptotic stability of the ImG.

The model of ImG along with the structure of local voltage and frequency controllers is presented in Section II. The passivity of ImG components and ImG stability are addressed in Section III. Simulations validating theoretical results are provided in Section IV. Finally, conclusions are drawn in Section V.

\section{A. Preliminaries and notation}

1) Sets, vectors, and functions: We let $\mathbb{R}$ (resp. $\mathbb{R}_{>0}$ ) denote the set of real (resp. strictly positive real) numbers. The notation $A \succ 0$ (resp. $A \succeq 0$ ) represents a positive definite (resp. positive semidefinite) matrix. Throughout, 0 is a matrix of all zeros of appropriate dimensions.

2) Passivity theory: Consider a control-affine nonlinear system

$$
\Sigma_{i}^{N L}=\left\{\begin{array}{l}
\dot{x}_{i}=q\left(x_{i}, u_{i}\right)=f\left(x_{i}\right)+g\left(x_{i}\right) u_{i}, \\
y_{i}=h_{i}\left(x_{i}\right)
\end{array},\right.
$$

where $x_{i} \in \mathbb{R}^{n_{i}}, y_{i} \in \mathbb{R}^{p}$, and $u_{i} \in \mathbb{R}^{p}$. The functions $q_{i}: \mathbb{R}^{n_{i}} \times \mathbb{R}^{p} \rightarrow \mathbb{R}^{n_{i}}, f: \mathbb{R}^{n_{i}} \rightarrow \mathbb{R}^{n_{i}}, g: \mathbb{R}^{n_{i}} \rightarrow \mathbb{R}^{n_{i}} \times \mathbb{R}^{p}$, and $h: \mathbb{R}^{n_{i}} \rightarrow \mathbb{R}^{p}$ are twice continuously differentiable, verifying $q_{i}(0,0)=0, f_{i}(0)=0$, and $h_{i}(0)=0$. Note that inputs and outputs have the same dimension $p$. The nonlinear system $\Sigma_{i}^{N L}$ is passive [17] if there exists a continuously differentiable positive-semidefinte storage function $\mathbf{V}_{i}\left(x_{i}\right) \geq$ $0, \mathbf{V}_{i}(0)=0$, and a function $\mathbf{S}_{i}\left(x_{i}\right)$, such that

$$
\dot{\mathbf{V}}_{i}\left(x_{i}\right)=u_{i}^{T} y_{i}-\mathbf{S}_{i}\left(x_{i}\right) \text {. }
$$

If $\mathbf{S}_{i}\left(x_{i}\right) \geq 0$, then system $\Sigma_{i}^{N L}$ is said to be passive. Moreover, the system $\Sigma_{i}^{N L}$ is strictly passive if $x_{i} \neq 0 \Rightarrow$ $\mathbf{S}_{i}\left(x_{i}\right)>0$. 
3) Skew-symmetric interconnections: Consider a set of $N$ subsystems with control-affine dynamics given by (1). Suppose that the subsystems are coupled together through the input

$$
u_{i}=\sum_{j \in \mathcal{N}_{i}^{+}} w_{i j} y_{j}-\sum_{j \in \mathcal{N}_{i}^{-}} w_{j i} y_{j} \quad i=1, \cdots, N
$$

where $w_{i j}$ are scalars. The interconnection between subsystems defined by (3) is called skew symmetric [18], [14].

\section{MICROGRID MODEL}

In this section, we describe the electric model of the islanded AC mirogrid (ImG) comprising of multiple distributed generation units (DGUs) connected to each other via power lines. In particular, we extend the model in [1], [19], [7], [8] by including line dynamics and allowing the loads to be connected to arbitrary network nodes. We assume threephase electrical signals without zero-sequence components and balanced network parameters.

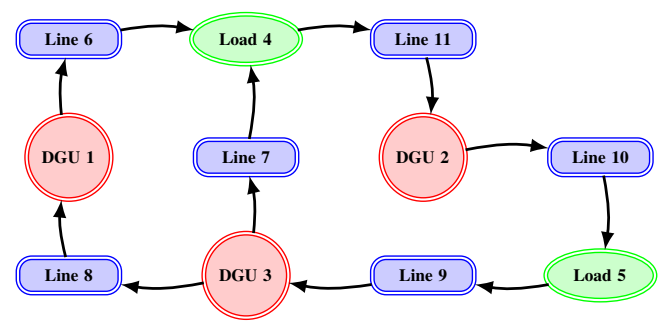

Fig. 1: A representative diagram of the $\mathrm{ImG}$ network. The sets $\mathcal{D}, \mathcal{L}$, and $\mathcal{P}$ are represented in red, green, and blue respectively.

ImG Model: The electric interconnections in an ImG are modeled as a directed connected graph $\mathcal{G}=(\mathcal{V}, \mathcal{E})$. $\mathcal{V}$ is partitioned into two sets: $\mathcal{M}=\{1, \ldots, n+m\}$ represents the DGUs and loads, and $\mathcal{P}=\{n+m+1, \cdots, m+n+o\}$ is the set of power lines. The set $\mathcal{M}$ is further divided into two sets: $\mathcal{D}=\{1, \ldots, n\}$ represents the DGUs and $\mathcal{L}=$ $\{n+1, \cdots, n+m\}$ the loads such that $\mathcal{M}=\mathcal{D} \cup \mathcal{L}$. Each DGU and load is interfaced with the ImG through a point of common coupling (PCC). We refer the reader to Figure 1 for a representative ImG diagram. The orientation of each edge represents the reference direction of positive currents which is arbitrarily assigned. It is evident that a line cannot have only in-neighbors or out-neighbors as the current entering in a line must leave it. Indeed, each node in $\mathcal{P}$ is always connected to two different nodes in $\mathcal{M}$ through two directed edges. We define a matrix $B \in \mathbb{R}^{N \times M}$, with DGUs and loads along rows and lines along columns, as

$$
B_{i l}:\left\{\begin{array}{rl}
1 & l \in \mathcal{N}_{i}^{+} \\
-1 & l \in \mathcal{N}_{i}^{-}, i \in \mathcal{M}, l \in \mathcal{N}, \\
0 & \text { otherwise }
\end{array}\right.
$$

where $\mathcal{N}_{i}^{+}=\{l \in \mathcal{V}:(i, l) \in \mathcal{E}\}$ denotes the set of out-neighbors, $\mathcal{N}_{i}^{-}=\{j \in \mathcal{V}:(j, i) \in \mathcal{E}\}$ the set of inneighbors, and $\mathcal{N}_{i}=\mathcal{N}_{i}^{+} \cup \mathcal{N}_{i}^{-}$the set of neighbors.

Remark 1: In order to facilitate control design, electrical signals in this work are represented in a $d q$ reference frame common to all DGUs rotating with speed $\omega_{0}$. This requires the controller clocks to be synchronized with sufficient precision. Technologies, requiring infrequent communication, for achieving this goal are available (see [8] or the review in [1]).

Dynamic model of a DGU: The single-phase equivalent scheme of DGU $i$ is shown in the left dashed frame of Figure 2 . The DGU comprises a DC voltage source (generally a renewable source), a voltage source converter (VSC), and a series $R L C$ filter. The $i^{t h} \mathrm{DGU}$ at $P C C_{i}$ is connected to other DGUs through power lines. Let $\omega_{0}$ be the reference network frequency. On applying Kirchhoff's current law (KCL) and Kirchoff's voltage law (KVL) on the DGU side at $P C C_{i}$, one obtains DGU model in $d q$ reference frame as (rotating with speed $\omega_{0}$ ) is

$$
\Sigma_{[i]}^{D G U}:\left\{\begin{array}{l}
\frac{\mathrm{d}}{\mathrm{d} t} V_{i}^{d q}=-\mathrm{i} \omega_{0} V_{i}^{d q}+\frac{I_{t i}^{d q}}{C_{t i}}-\frac{I_{i}^{d q *}}{C_{t i}} \\
\frac{\mathrm{d}}{\mathrm{d} t} I_{t i}^{d q}=-\left(\frac{R_{t i}}{L_{t i}}+\mathrm{i} \omega_{0}\right) I_{t i}^{d q}-\frac{V_{i}^{d q}}{L_{t i}}+\frac{V_{t i}^{d q}}{L_{t i}}
\end{array}, i \in \mathcal{D},\right.
$$

where $I_{i}^{*}$, a function of line currents, is the net-current injected into the $\mathrm{DCmG}$ and is given by

$$
I_{i}^{d q *}=\sum_{l \in \mathcal{N}_{i}^{+}} B_{i l} I_{l}^{d q}+\sum_{l \in \mathcal{N}_{i}^{-}} B_{i l} I_{l}^{d q}=\sum_{l \in \mathcal{N}_{i}} B_{i l} I_{l}^{d q} .
$$

In (5), quantities $V_{i}^{d q}$ and $I_{t i}^{d q}$ represent the $i$-th PCC voltage and filter current, respectively, $V_{t i}^{d q}$ is the command input to the corresponding VSC, while the terms $R_{t i} \in \mathbb{R}_{>0}$, $L_{t i} \in \mathbb{R}_{>0}$, and $C_{t i} \in \mathbb{R}_{>0}$ are the internal resistance, capacitance (lumped with the line capacitances), and inductance of the DGU converter. We remark that $d q$ quantities in (5) are complex variables. One can deduce the state-space representation of the DGU model by separating the real and imaginary part as dynamics (5). Notably, we can write

$$
\Sigma_{[i]}^{D G U}:\left\{\begin{array}{l}
\dot{x}_{[i]}(t)=A_{i i} x_{[i]}(t)+B_{i}^{D} u_{[i]}^{D}(t)+B_{i} u_{[i]}(t) \\
y_{[i]}(t)=C_{i} x_{[i]}(t)
\end{array}, i \in \mathcal{D},\right.
$$

where $x_{[i]}=\left[V_{i}^{d}, V_{i}^{q}, I_{t i}^{d}, I_{t i}^{q}\right]^{T}$ is the state, $y_{[i]}=\left[V_{i}^{d}, V_{i}^{q}\right]^{T}$ is the output, $u_{[i]}^{D}=\left[V_{t i}^{d}, V_{t i}^{q}\right]^{T}$ is the internal control input to the DGU and $u_{[i]}=\left[-I_{i}^{d *},-I_{i}^{q *}\right]^{T}$ is the coupling input. We highlight that $u_{[i]}$ accounts for the coupling with the rest of the microgrid. The matrices in (7) have the following form:

$$
A_{i i}=\left[\begin{array}{cccc}
0 & \omega_{0} & \frac{1}{C_{t i}} & 0 \\
-\omega_{0} & 0 & 0 & \frac{1}{C_{t i}} \\
-\frac{1}{L_{t i}} & 0 & -\frac{R_{t i}}{L_{t i}} & \omega_{0} \\
0 & -\frac{1}{L_{t i}} & -\omega_{0} & -\frac{R_{t i}}{L_{t i}}
\end{array}\right], B_{i}^{D}=\left[\begin{array}{cc}
\mathbf{0} & \mathbf{0} \\
\frac{1}{L_{t i}} & 0 \\
0 & \frac{1}{L_{t i}}
\end{array}\right] \text {, }
$$

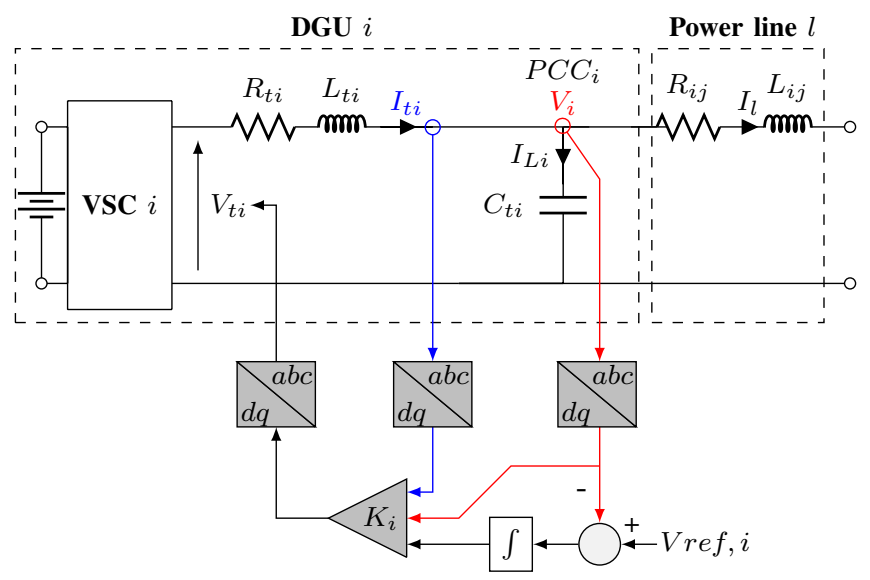

Fig. 2: Electrical scheme of DGU $i$, power line $l$, and local PnP voltage and frequency controller. 


$$
B_{i}=\left[\begin{array}{cc}
\frac{1}{C_{t i}} & 0 \\
0 & \frac{1}{C_{t i}} \\
\mathbf{0} & \mathbf{0}
\end{array}\right], \text { and } C_{i}=\left[\begin{array}{ll}
I_{2} & \mathbf{0}
\end{array}\right]
$$

Load model: The $j^{\text {th }}$ load along with connecting power lines is shown in Figure 3. On applying $\mathrm{KCL}$ at $P C C_{i}$, one obtains the dynamic model of the load in $d q$ frame as

$\Sigma_{[j]}^{L \text { ood }}:\{\frac{\mathrm{d}}{\mathrm{d} t} V_{j}^{d q}=-\mathrm{i} \omega_{0} V_{j}^{d q}-\frac{1}{C_{t j}} \underbrace{\left(Y_{L j} V_{j}^{d q}+\bar{I}_{L j}^{d q}\right)}_{I_{L j}}-\frac{I_{i}^{d q *}}{C_{t j}}, j \in \mathcal{L}$

where $Y_{L j}, \bar{I}_{L j}^{d q}$, and $I_{L j}$ denote load impedance, constant load current, and total load current respectively. One can write (8) in state-space form as

$$
\Sigma_{[j]}^{L \text { oad }}:\left\{\begin{array}{l}
\dot{x}_{[j]}(t)=A_{j j} x_{[j]}(t)+M_{j} d_{[j]}(t)+B_{j} u_{[j]}(t) \\
y_{[j]}(t)=C_{j} x_{[j]}(t)
\end{array}, j \in \mathcal{L},\right.
$$

where $x_{[j]}=\left[V_{j}^{d}, V_{j}^{q}\right]^{T}$ is the state, $y_{[j]}=\left[V_{j}^{d}, V_{j}^{q}\right]^{T}$ is the output, $d_{[j]}=\left[I_{L j}^{d}, I_{L j}^{q}\right]^{T}$ the exogenous input, and

$$
u_{[j]}=\left[-I_{j}^{d *},-I_{j}^{q *}\right]^{T}
$$

is the coupling input. The matrices in (9) are as follows:

$$
\begin{aligned}
A_{j j} & =\left[\begin{array}{cc}
-\frac{Y_{L j}}{C_{t j}} & \omega_{0} \\
-\omega_{0} & -\frac{Y_{L j}}{C_{t j}}
\end{array}\right], B_{j}=\left[\begin{array}{cc}
\frac{1}{C_{t j}} & 0 \\
0 & \frac{1}{C_{t j}}
\end{array}\right], \\
M_{j} & =I_{2}, \text { and } C_{j}=I_{2} .
\end{aligned}
$$

Dynamic model of a power line: The power lines are rep-

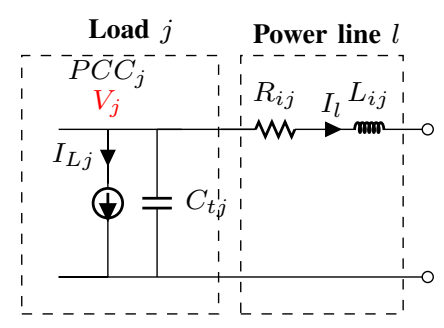

Fig. 3: Electrical scheme of ZI load. The current $I_{L j}$ incorporates the effect of load impedance $Y_{L j}$.

resented by the $\pi$-equivalent model of the transmission line [20]. On the DGU side, it is assumed that the line capacitance is lumped with the DGU filter capacitance (capacitor $C_{t i}$ in Figure 2). Similarly, on the load side of the line, its capacitance is embedded into the load capacitance as shown in Figure 3. Therefore, as shown in Figure 2, the power line $l$ is modeled as an $R L$ circuit with resistance $R_{l}>0$ and inductance $L_{l}>0$. By applying KVL on the $l^{t h}$ power line, one obtains

$$
\Sigma_{[l]}^{L i n e}:\left\{\frac{\mathrm{d}}{\mathrm{d} t} I_{l}^{d q}=-\left(\frac{R_{t l}}{L_{t l}}+\mathrm{i} \omega_{0}\right) I_{l}^{d q}+\frac{1}{L_{l}} \sum_{i \in \mathcal{N}_{l}} B_{i l} V_{i}^{d q}, l \in \mathcal{P}\right.
$$

where the variables $V_{i}$ and $I_{l}$ represent the voltage at $P C C_{i}$ and the current flowing through the $l^{\text {th }}$ power line respectively. One can write (12) in state-space form as

$$
\Sigma_{[l]}^{\text {Line }}:\left\{\begin{array}{l}
\dot{x}_{[l]}(t)=A_{l l} x_{[l]}(t)+B_{l} u_{[l]}(t) \\
y_{[l]}(t)=C_{l} x_{[l]}(t)
\end{array}, l \in \mathcal{P}\right.
$$

where $x_{[l]}=\left[I_{l}^{d}, I_{l}^{q}\right]^{T}$ is the state, $y_{[l]}=\left[I_{l}^{d}, I_{l}^{q}\right]^{T}$ is the output, and

$$
u_{[l]}=\sum_{k \in \mathcal{N}_{l}} B_{k l}\left[V_{k}^{d}, V_{k}^{q}\right]^{T}=\sum_{k \in \mathcal{N}_{l}} B_{k l} y_{[k]}, k \in \mathcal{M}
$$

is the coupling input. The matrices in (13) are as follows:

$$
A_{l l}=\left[\begin{array}{cc}
-\frac{R_{t l}}{L_{t l}} & \omega_{0} \\
-\omega_{0} & -\frac{R_{t l}}{L_{t l}}
\end{array}\right], B_{l}=\left[\begin{array}{cc}
\frac{1}{L_{t l}} & 0 \\
0 & \frac{1}{L_{t l}}
\end{array}\right], \text { and } C_{l}=I_{2}
$$

\section{A. Structure of local controllers}

As in [7], the main objective of a DGU is to track constant references $\mathbf{y}_{\text {ref }}(t)=\overline{\mathbf{y}}_{\text {ref }}$ at its PCC. These references are assumed to be provided by a higher-level controller or through an entity regulating the grid. To this aim, the DGUs model is augmented with integrators (see Figure 2, where $\left.y_{\text {ref } f_{[i]}}=V_{r e f, i}\right)$. Hence, we first write the dynamics of the integrators as

$$
\begin{aligned}
\dot{v}_{[i]}(t)=e_{[i]}(t) & =y_{\operatorname{ref}[i]}(t)-y_{[i]}(t) \\
& =y_{\operatorname{ref}[i]}(t)-C_{i} x_{[i]}(t)
\end{aligned}, i \in \mathcal{D}
$$

and subsequently equip each DGU with a state-feedback controller

$$
\mathcal{C}_{[i]}: \quad u_{[i]}^{D}(t)=K_{i} \hat{x}_{[i]}(t),
$$

where $\hat{x}_{[i]}=\left[x_{[i]}^{T}, v_{[i]}^{T}\right]^{T} \in \mathbb{R}^{6}$ is the augmented state of the $i^{\text {th }}$ DGU and $K_{i}=\mathbb{R}^{2 \times 6}$ is the matrix collecting the control gains. We now derive the augmented model of the DGU as

$\hat{\Sigma}_{[i]}^{D G U}:\left\{\begin{array}{l}\dot{\hat{x}}_{[i]}(t)=\underbrace{\left(\hat{A}_{i i}+\hat{B}_{i}^{D} K_{i}\right)}_{F_{i}} \hat{x}_{[i]}(t)+\hat{M}_{i} \hat{d}_{[i]}(t)+\hat{B}_{i} \hat{u}_{[i]} \\ \hat{y}_{[i]}(t)=\hat{C}_{i} \hat{x}_{[i]}(t)\end{array}, i \in \mathcal{D}\right.$

where $\hat{y}_{[i]}=y_{[i]}$ is the output, $\hat{d}_{[i]}=\left[V_{r e f, i}^{d}, V_{r e f, i}^{q}\right]^{T} \in \mathbb{R}^{2}$ represents the exogenous signals, and

$$
\hat{u}_{[i]}=u_{[i]}=\left[-I_{i}^{d *},-I_{i}^{q *}\right]^{T}=\sum_{l \in \mathcal{N}_{i}} B_{i l}\left[I_{l}^{d},-I_{l}^{q}\right]^{T}=\sum_{l \in \mathcal{N}_{i}} B_{i l} y_{[l]} .
$$

Moreover, matrices in (17) have the form

$$
\begin{aligned}
\hat{A}_{i i} & =\left[\begin{array}{c|c}
A_{i i} & \mathbf{0} \\
\hline-C_{i} & \mathbf{0}
\end{array}\right]=\left[\begin{array}{cc|c}
\hat{\mathcal{A}}_{11, i} & \frac{1}{C_{t i}} I_{2} & \mathbf{0}_{2} \\
-\frac{1}{L_{t i}} I_{2} & \hat{\mathcal{A}}_{22, i} & \mathbf{0}_{2} \\
\hline-I_{2} & \mathbf{0}_{2} & \mathbf{0}_{2}
\end{array}\right], \\
\hat{\mathcal{A}}_{11, i} & =\omega_{0}\left[\begin{array}{cc}
0 & 1 \\
-1 & 0
\end{array}\right], \quad \hat{\mathcal{A}}_{22, i}=\left[\begin{array}{cc}
-\frac{R_{t i}}{L_{t i}} & \omega_{0} \\
-\omega_{0} & -\frac{R_{t i}}{L_{t i}}
\end{array}\right], \\
\hat{B}_{i}^{D} & =\left[\begin{array}{c}
B_{i}^{D} \\
\mathbf{0}
\end{array}\right], \hat{B}_{i}=\left[\begin{array}{c}
B_{i} \\
\mathbf{0}
\end{array}\right] \hat{C}_{i}=\left[\begin{array}{ll}
C_{i} & \mathbf{0}
\end{array}\right], \quad \hat{M}_{i}=\left[\begin{array}{c}
\mathbf{0} \\
I_{2}
\end{array}\right] .
\end{aligned}
$$

By setting $K_{i}=\left[\begin{array}{lll}\mathcal{K}_{11, i} & \mathcal{K}_{12, i} & \mathcal{K}_{13, i}\end{array}\right], \mathcal{K}_{1 j, i} \in \mathbb{R}^{2 \times 2}, j=$ $1,2,3$, the matrix $F_{i}$ in (17) is obtained as

$$
F_{i}=\left[\begin{array}{ccc}
\hat{\mathcal{A}}_{11, i} & \frac{1}{C_{t i}} I_{2} & \mathbf{0}_{2} \\
-\frac{1}{L_{t i}}\left(I_{2}-\mathcal{K}_{11, i}\right) & \hat{\mathcal{A}}_{22, i}+\frac{1}{L_{t i}}\left(\mathcal{K}_{12, i}\right) & \frac{1}{L_{t i}} \mathcal{K}_{13, i} \\
-I_{2} & \mathbf{0}_{2} & \mathbf{0}_{2}
\end{array}\right]
$$

As shown in [7], the pair $\left(\hat{A}_{i i}, \hat{B}_{i}^{D}\right)$ is always controllable and therefore, (17) can be stabilized. In addition, the control architecture induced by (16) is decentralized.

Remark 2 (Decoupled $d$ - $q$ control): The control-gain matrix $K_{i}$ maps the state $\hat{x}_{[i]}$ to the internal control input $u_{[i]}^{D}=$ $\left[V_{t i}^{d}, V_{t i}^{q}\right]^{T}$ of of $\hat{\Sigma}_{[i]}^{D G U}$. If the sub-matrices $\mathcal{K}_{1 j, i} \in \mathbb{R}^{2 \times 2}$, $j=1,2,3$ of $K_{i}$ are diagonal, $V_{t i}^{d}$ depends only upon the d-components of $\hat{x}_{[i]}$ and $V_{t i}^{q}$ only on the q-components. In such a case, the controller $\mathcal{C}_{[i]}$ is said to be decoupled. 


\section{APPLICATION OF PASSIVITY THEORY TO MICROGRIDS}

The ImG is a networked system defined by the mutual interconnection of its various subsytems i.e., loads (9), power lines (13), and DGUs (17). For the input-output pairs defined in (9), (13), and (17), one can use Theorem 1 in [14], relying on skew-symmetric interconnections defined in [18], to characterize the asymptotic behavior of ImG states and guarantee its stability. However, two necessary preconditions must be satisfied:

C.1 the ImG subsystems must be connected in a skewsymmetric fashion defined by (3), and

C.2 each and every subsystem must be passive.

Hereafter, we will discuss the validity of these preconditions in the ImG. For the condition C.1, we have the following result.

Lemma 1: The electrical interconnections in $\mathrm{DCmG}$ between DGUs, loads, and power lines given by (18), (10) and (14) are skew-symmetric.

Proof: The input-output relations in the ImG defined by (10), (14), and (18) are skew symmetric (see (3)) with $w_{i j}=$ $-1,(i, j) \in \mathcal{E}$. The proof is similar to the one provided in [14] and is omitted due to space constraints.

We will now discuss condition C.2, that is, the passivity of the various ImG subsystems. Before proceeding, the reader is reminded that the vectors $d_{[i]}$ and $d_{[j]}$ in equations (17) and (9) respectively, vary for different choices of constant exogenous inputs $V_{\text {ref,i }}$ and $\bar{I}_{L j}^{d q}$. Since the equations (9), (13), and (17) defining the overall ImG dynamics are linear, the stability of equilibrium in the absence of $d_{[i]}$ and $d_{[j]}$ is equivalent to the stability of all the equilibria generated for different (yet constant) $d_{[i]}$ and $d_{[j]}$. Therefore, in the ensuing sections, where we study the passivity of the various ImG subsystems, we set $d_{[i]}$ and $d_{[j]}$ to zero. The passivity of loads and power lines are summarized in the following remarks.

Remark 3 (Passivity of power lines): As shown in [21], RL power lines in (13) are always strictly passive with a positive-definite storage function

$$
\mathbf{V}_{[l]}\left(x_{[l]}\right)=\frac{1}{2} x_{[l]}^{T} L_{l} I_{2} x_{[l]} \text { and } \mathbf{S}_{[l]}\left(x_{[l]}\right)=x_{[l]}^{T} R_{l} I_{2} x_{[l]}, l \in \mathcal{P},
$$

where $I_{2}$ is an identity matrix.

Furthermore, the loads in (9) are strictly passive with a positive-definite storage function

$$
\mathbf{V}_{[l]}\left(x_{[j]}\right)=\frac{1}{2} x_{[j]}^{T} C_{t j} I_{2} x_{[j]} \text { and } \mathbf{S}_{[j]}\left(x_{[j]}\right)=x_{[j]}^{T} Y_{L j} I_{2} x_{[j]}, j \in \mathcal{L} \text {. }
$$

The loads can cease to be strictly passive when the load admittance $Y_{L j}=0$. In such a scenario, $\mathbf{S}_{[j]}\left(x_{[j]}\right)=0$.

\section{A. Passivity of the $D G U$}

Given the passivity of lines and loads, the next step is to show that the DGUs can by passivated by appropriately choosing the control gains. To this aim, we propose the candidate storage function

$$
\mathbf{V}_{[i]}\left(\hat{x}_{[i]}\right)=\frac{1}{2} \hat{x}_{[i]}^{T} P_{i} \hat{x}_{[i]}, i \in \mathcal{D}
$$

where $P_{i}=P_{i}^{T} \in \mathbb{R}^{6 \times 6}$ is a positive definite matrix. The following theorem reveals that $P_{i}$ must satisfy additional structural constraints in order to passivate $\hat{\Sigma}_{i}^{D G U}$. Moreover, we show that decoupled control gains (see Remark 2) can never render a DGU passive.

Theorem 1: Consider the closed-loop DGU model in (17) with $d_{[i]}=0$. For the defined input-output pair, the following statements hold:

I) The DGU is passive if and only if passivity can be certified by the storage function (23) with $P_{i} \succ 0$ conforming to the following structure

$$
P_{i}=\left[\begin{array}{c|cc}
C_{t i} I_{2} & \mathbf{0} & \mathbf{0} \\
\hline \mathbf{0}_{2} & \mathcal{X}_{i}+\mathcal{X}_{i} \mathcal{Z}_{i} \mathcal{X}_{i} & -\mathcal{X}_{i} \mathcal{Z}_{i} \\
\mathbf{0}_{2} & -\mathcal{Z}_{i} \mathcal{X}_{i} & \mathcal{Z}_{i}
\end{array}\right],
$$

where $\mathcal{Z}_{i}, \mathcal{X}_{i} \in \mathbb{R}^{2 \times 2}$ are positive definite matrices.

II) The DGU can never be strictly passive.

III) Decoupled d-q control can not render the DGU passive.

Proof: Let $P_{i} \succ 0$ be partitioned into $2 \times 2$ square matrices as

$$
P_{i}=\left[\begin{array}{lll}
\mathcal{P}_{11, i} & \mathcal{P}_{12, i} & \mathcal{P}_{13, i} \\
\mathcal{P}_{12, i}^{T} & \mathcal{P}_{22, i} & \mathcal{P}_{23, i} \\
\mathcal{P}_{13, i}^{T} & \mathcal{P}_{23, i}^{T} & \mathcal{P}_{33, i}
\end{array}\right]
$$

The DGU (17) is a linear time-invariant system. Therefore, the following conditions, given by the KYP Lemma [21], are necessary and sufficient for the passivity of the DGU:

$$
\begin{gathered}
\left(\hat{A}_{i i}+\hat{B}_{i} K_{i}\right)^{T} P_{i}+P_{i}\left(\hat{A}_{i i}+\hat{B}_{i} K_{i}\right)=Q_{i} \\
P_{i} \hat{B}_{i}=\hat{C}_{i}^{T}
\end{gathered}
$$

where the matrix

$$
Q_{i} \preceq 0 .
$$

Moreover, for a DGU to be strictly passive, one must have $Q_{i} \prec 0$. By direct calculation, it can be verified that $(26 \mathrm{~b})$ holds when $\mathcal{P}_{12, i}=\mathcal{P}_{13, i}=0$ and $\mathcal{P}_{11, i}=C_{t i} I_{2}$. Therefore, $P_{i}$ must be of the form

$$
P_{i}=\left[\begin{array}{c|c}
C_{t i} I_{2} & \mathbf{0} \\
\hline \mathbf{0} & P_{22, i}
\end{array}\right], P_{22, i} \in \mathbb{R}^{4 \times 4} .
$$

We highlight that $Q_{i}$ depends upon matrices $P_{i}$ and $K_{i}$, which must be chosen in an appropriate way in order to comply with (27). Following the approach in [1], let us parametrize the unknown quantities in (26a) as follows

$$
P_{i}=Y_{i}^{-1}, \quad K_{i}=G_{i} Y_{i}^{-1},
$$

where $G_{i}=\left[\begin{array}{lll}\mathcal{G}_{11, i} & \mathcal{G}_{12, i} & \mathcal{G}_{13, i}\end{array}\right] \in \mathbb{R}^{2 \times 6}, \mathcal{G}_{11, i}, \mathcal{G}_{12, i}, \mathcal{G}_{13, i} \in$ $\mathbb{R}^{2 \times 2}$,

$$
Y_{i}=\left[\begin{array}{c|cc}
C_{t i}^{-1} I_{2} & \mathbf{0}_{2} & \mathbf{0}_{2} \\
\hline \mathbf{0}_{2} & \mathcal{Y}_{22, i} & \mathcal{Y}_{23, i} \\
\mathbf{0}_{2} & \mathcal{Y}_{23, i}^{T} & \mathcal{Y}_{33, i}
\end{array}\right]=\left[\begin{array}{c|c}
C_{t i}^{-1} I_{2} & \mathbf{0} \\
\hline \mathbf{0} & Y_{22, i}
\end{array}\right],
$$

and

$$
\begin{gathered}
\mathcal{G}_{11, i}=C_{t i}^{-1} \mathcal{K}_{11, i} \\
\mathcal{G}_{12, i}=\mathcal{K}_{12, i} \mathcal{Y}_{22, i}+\mathcal{K}_{13, i} \mathcal{Y}_{23, i}^{T} \\
\mathcal{G}_{13, i}=\mathcal{K}_{12, i} \mathcal{Y}_{23, i}+\mathcal{K}_{13, i} \mathcal{Y}_{33, i} .
\end{gathered}
$$

Using (29), (26a) can be equivalently written as

$$
Y_{i} \hat{A}_{i i}^{T}+G_{i}^{T} \hat{B}_{i}+\hat{A}_{i i} Y_{i}+\hat{B}_{i} G_{i}=\tilde{Q}_{i}
$$

where

$$
\tilde{Q}_{i}=Y_{i} Q_{i} Y_{i}
$$

To ensure passivity of DGU (17), (27) must hold. Since $Y_{i} \succ$ 0 , one has that $Q_{i} \preceq 0$ only if $\tilde{Q}_{i}$ has the same property. In 
the remainder of the proof we will reason on the negative semi-definiteness of $\tilde{Q}_{i}$.

As shown in [1], $\tilde{Q}_{i}$ is negative semidefinite if the matrix inequality

$$
\hat{\mathcal{A}}_{22, i} \mathcal{Y}_{22, i}+\mathcal{Y}_{22, i} \hat{\mathcal{A}}_{22, i}^{T}+\frac{1}{L_{t i}} \mathcal{G}_{12, i}+\frac{1}{L_{t i}} \mathcal{G}_{12, i}^{T} \preceq 0,
$$

and the following equalities

$$
\begin{gathered}
\mathcal{Y}_{23, i}=I_{2}, \\
\mathcal{G}_{13, i}=-L_{t i} \hat{\mathcal{A}}_{22, i}, \\
\mathcal{G}_{11, i}=\frac{L_{t i}}{C_{t i}} \mathcal{Y}_{22, i}+\frac{1}{C_{t i}} I_{2},
\end{gathered}
$$

hold simultaneously. Moreover, using Schur's complement [22], one has that the matrix $Y_{i} \succ 0$ if and only if $\mathcal{Y}_{22, i} \succ 0$ and

$$
\mathcal{Y}_{33, i}-\mathcal{Y}_{23, i}^{T} \mathcal{Y}_{22, i}^{-1} \mathcal{Y}_{23, i}=\mathcal{Y}_{33, i}-\mathcal{Y}_{22, i}^{-1} \succ 0 .
$$

One can equivalently parametrize $Y_{33, i}$ as

$$
\mathcal{Y}_{33, i}=\mathcal{Y}_{22, i}^{-1}+\mathcal{Y}_{i}
$$

where $\mathcal{Y}_{i} \succ 0 \in \mathbb{R}^{2 \times 2}$. We will now show that if (35) and (37) hold, (34a) is always solvable. Using (31), (35), and (37), the inequality (34a) can be simplified as

$$
\begin{aligned}
& \hat{\mathcal{A}}_{22, i} \mathcal{Y}_{22, i}+\mathcal{Y}_{22, i} \hat{\mathcal{A}}_{22, i}^{T}+\frac{1}{L_{t i}} \mathcal{G}_{12, i}+\frac{1}{L_{t i}} \mathcal{G}_{12, i}^{T} \\
& =-\mathcal{K}_{13, i}\left(\mathcal{Y}_{i} \mathcal{Y}_{22, i}\right)-\left(\mathcal{Y}_{i} \mathcal{Y}_{22, i}\right)^{T} \mathcal{K}_{13, i}^{T} \preceq 0
\end{aligned}
$$

The last equation is always solvable: one particular solution is $\mathcal{K}_{13, i}=\left(\mathcal{Y}_{i} \mathcal{Y}_{22, i}\right)^{-1}$. Indeed, the matrices $Y_{i}$ and $\mathcal{Y}_{22, i}$ are positive definite, and by Sylvester's law of inertia [22], $\left(\mathcal{Y}_{i} \mathcal{Y}_{22, i}\right)$ is always invertible. Using (35) and (36),

$$
Y_{i}=\left[\begin{array}{c|cc}
C_{t i}^{-1} I_{2} & \mathbf{0}_{2} & \mathbf{0}_{2} \\
\hline \mathbf{0}_{2} & \mathcal{Y}_{22, i} & I \\
\mathbf{0}_{2} & I & \mathcal{Y}_{22, i}^{-1}+\mathcal{Y}_{i}
\end{array}\right] .
$$

Note that $Y_{i}$ must conform to the above structure in order to guarantee the negative semi-definiteness of $\tilde{Q}_{i}$ and subsequently of $Q_{i}$. The $P_{i}$ in (24) is nothing but the inverse of $Y_{i}$ with $\mathcal{X}_{i}=\mathcal{Y}_{22, i}^{-1}$ and $\mathcal{Z}_{i}=\mathcal{Y}_{i}$. This concludes the proof of statement I).

For proving Statement II), assume that $Q_{i}$ is partitioned in a similar fashion as (25). On evaluating the left-hand side of (26a) with $P_{i}$ in (24), one obtains that $\mathcal{Q}_{11, i}=0$. Since the first minor of $Q_{i}$ is zero, it can never be negative definite. Hence, the DGU can never be strictly passive.

We will focus on (31c) to prove Statement III). Using (35a) and (35b), it can rewritten as

$$
-\mathcal{K}_{13, i}^{-1}\left(L_{t i} \hat{\mathcal{A}}_{22, i}+\mathcal{K}_{12, i}\right)=\mathcal{Y}_{33, i}
$$

Under decoupled control, the matrices $\mathcal{K}_{12, i}$ and $\mathcal{K}_{13, i}$ are diagonal. By using $\hat{\mathcal{A}}_{22, i}$ defined in (19), it can be shown that the left hand side of the above equation has unequal offdiagonal elements and hence, fails to be symmetric. Since $\mathcal{Y}_{33, i}$ is symmetric, decoupled dq control cannot passivate the DGUs.

For $P_{i}$ in (24), one can verify that the derivative of $\mathbf{V}_{[i]}\left(x_{[i]}\right)$ along the state trajectory of $\hat{\Sigma}_{i}^{D G U}$ in (17) is obtained as

$$
\dot{\mathbf{V}}_{[i]}\left(\hat{x}_{[i]}\right)=u_{[i]}^{T} y_{[i]}-\underbrace{\hat{x}_{[i]}^{T}\left(-Q_{i}\right) \hat{x}_{[i]}}_{\mathbf{S}_{[i]}\left(x_{[i]}\right)},
$$

where

$$
Q_{i}=\left(\hat{A}_{i i}+\hat{B}_{i} K_{i}\right)^{T} P_{i}+P_{i}\left(\hat{A}_{i i}+\hat{B}_{i} K_{i}\right) .
$$

Furthermore, passivity of the DGU corresponds to $Q_{i} \preceq$ 0 . As shown in Theorem 1, this condition can always be fulfilled for suitable control gains $K_{i}$. The problem of computing $K_{i}$ for a given choice of $P_{i}$ can be cast into a linear matrix inequality and is solved using numerical optimization. We refer the reader to [1] for further details.

\section{B. Asymptotic stability of the microgrid}

As shown in Lemma 1, the interconnections among the various ImG subsystems are skew-symmetric. In addition, the power lines are strictly passive, the loads are passive, and the DGUs can be passivated using suitable gains $K_{i}$. Hence, both the conditions $\mathrm{C} 1$ and $\mathrm{C} 2$ are satisfied. At this point, one can prove the asymptotic stability of the ImG network.

Theorem 2: If $\hat{\Sigma}_{[i]}^{D G U}$ in (17) is passive for all $i \in \mathcal{D}$, then the origin of ImG, defined by the interconnection of (9), (13), and (17), is asymptotically stable.

Proof: Given the passivity of the DGUs, the conditions C.1 and C.2 hold simultaneously. From [14, Theorem 1], one can conclude that the ImG states asymptotically converge to the largest invariant set contained in

$$
\begin{array}{r}
E=\left\{\hat{x}_{[i]}, x_{[j]}, x_{[l]}: \mathbf{S}_{[i]}\left(\hat{x}_{[i]}\right)=0, \mathbf{S}_{[j]}\left(x_{[j]}\right)=0,\right. \\
\left.\mathbf{S}_{[l]}\left(x_{[l]}\right)=0, i \in \mathcal{D}, j \in \mathcal{L}, l \in \mathcal{P}\right\} .
\end{array}
$$

Since the DGUs are never strictly passive, the set $E$ is never empty. However, one can show that the largest invariant set in $E$ is the origin and hence, the origin of the $\operatorname{ImG}$ is asymptotically stable. The derivation of the largest invariant is similar to the one provided in [1] and is omitted due space constraints.

Remark 4 (PnP nature of control): From (42), for computing $K_{i}$ such that $Q_{i}$ is negative semidefinite, one needs the knowledge of matrix $\hat{A}_{i i}$. It should be noted that $\hat{A}_{i i}$ depends only on the filter parameters of the $i^{\text {th }}$ DGU. Therefore, controller $\mathcal{C}_{[i]}$ can be synthesized in a purely decentralized fashion. We defer the reader to [1] for detailed comments about the benefits of this PnP design procedure, which facilitates plug-and-play operations, that is, addition and removal of the DGUs on the fly by designing local controllers at a computational cost independent of the size of ImG.

\section{Simulation Results}

In this section, we aim to validate the proposed theoretical arguments for analyzing the stability of an ImG through simulation studies. We consider an ImG composed of 2 DGU nodes with non identical parameters and 4 load nodes (see Figure 4). The reader is referred to [7] for parameter values used in the simulation.In particular, the frequency used for $d q$ transformation is $\omega_{0}=50 \mathrm{~Hz}$. In our experiments, voltage references $V_{\text {ref,1 } 1}, V_{\text {ref,2 }}$ are assumed to be provided by a higher level controller. In order to design our controllers, we use a matrix $P_{i}$ (see (24)) with $\mathcal{X}_{i}=\mathcal{Z}_{i}=I_{2}$. We initially provide a voltage reference $V_{1, \text { ref }}^{d}=1.05 \mathrm{pu}$, $V_{1, \text { ref }}^{q}=0.96 \mathrm{pu}$, and $V_{5, \text { ref }}^{q}=1.07 \mathrm{pu}, V_{5, \text { ref }}^{1}=1.02 \mathrm{pu}$, which is changed $V_{1, \text { ref }}^{d}=1.01 \mathrm{pu}, V_{1, \text { ref }}^{q}=1.05 \mathrm{pu}$, and 


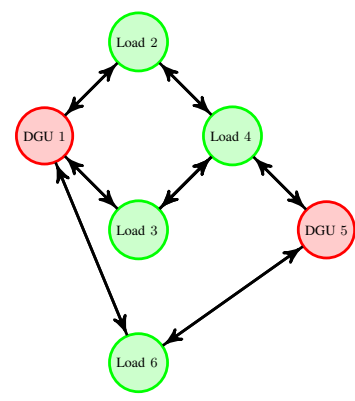

Fig. 4: Simplified representation ImG composed of 2 DGUs and 4 load nodes. The connecting power lines are represented by the edges.

$V_{5, \text { ref }}^{q}=1.02 \mathrm{pu}, V_{5, \text { ref }}^{1}=0.96 \mathrm{pu}$ at $t=10 \mathrm{~s}$. As shown in Figure 5, the voltages exhibit small deviations at $t=10$ $\mathrm{s}$ but are promptly restored desired reference values by the control actions. This shows the local controllers can stabilize the entire network. Although not shown here, when the off-
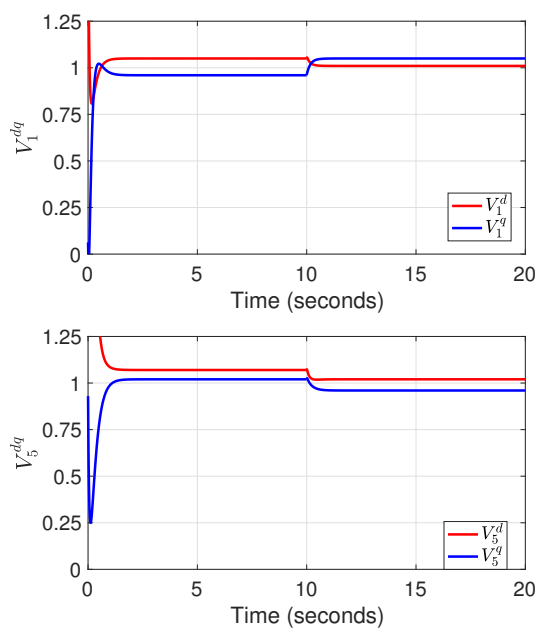

Fig. 5: Components $d$ (red) and $q$ (blue) of DGU voltages.

diagonal terms of sub-matrices $\mathcal{K}_{11, i}, \mathcal{K}_{12, i}$, and $\mathcal{K}_{12, i}$ of $K_{i}$ are neglected, we noticed that the voltages do not converge, the ImG stability is lost.

\section{CONClusion}

In this work, we dealt with the problem of voltage and frequency stability in ImG. An ImG model with ZI loads appearing at generic positions and dynamic RL lines was considered. We showed that the stability of the ImG hinges on the passivity of its various subsystems. For the DGUs, we provide necessary and sufficient conditions for the existence of control gains guaranteeing passivity. Moreover, the passivating control design is completely decentralized, and is independent of the ImG size and topology.

Many interesting future research directions can be taken. An immediate next step can be the inclusion of load models more sophisticated than the ZI models considered in this work. Designing the interface between local regulators and higher-level control layers for efficient operation of the entire ImG is an other promising direction.

\section{REFERENCES}

[1] M. Tucci and G. Ferrari-Trecate, "A scalable, line-independent control design algorithm for voltage and frequency stabilization in AC islanded microgrids," Tech. Rep., 2018. [Online]. Available: https://arxiv.org/abs/1703.02336

[2] J. M. Guerrero, M. Chandorkar, T. Lee, and P. C. Loh, "Advanced Control Architectures for Intelligent Microgrids Part I: Decentralized and Hierarchical Control," IEEE Transactions on Industrial Electronics, vol. 60, no. 4, pp. 1254-1262, 2013.

[3] T. Dragicevic, X. Lu, J. C. Vasquez, and J. M. Guerrero, "DC microgrids part 1: A review of control strategies and stabilization techniques," IEEE Transactions on Power Electronics, vol. 31, no. 7, pp. 4876-4891, 2016.

[4] J. M. Guerrero, J. C. Vsquez, and R. Teodorescu, "Hierarchical control of droop-controlled DC and AC microgrids: a general approach towards standardization," in 2009 35th Annual Conference of IEEE Industrial Electronics, 2009, pp. 4305-4310.

[5] J. Schiffer, R. Ortega, A. Astolfi, J. Raisch, and T. Sezi, "Conditions for stability of droop-controlled inverter-based microgrids," Automatica, vol. 50, no. 10, pp. 2457-2469, 2014.

[6] J. W. Simpson-Porco, F. Dörfler, and F. Bullo, "Synchronization and power sharing for droop-controlled inverters in islanded microgrids," Automatica, vol. 49, no. 9, pp. 2603-2611, 2013.

[7] S. Riverso, F. Sarzo, and G. Ferrari-Trecate, "Plug-and-play voltage and frequency control of islanded microgrids with meshed topology," IEEE Transactions on Smart Grid, vol. 6, no. 3, pp. 1176-1184, 2015.

[8] A. H. Etemadi, E. J. Davison, and R. Iravani, "A decentralized robust control strategy for multi-DER microgrids part 1: Fundamental concepts," IEEE Transactions on Power Delivery, vol. 27, no. 4, pp. 1843-1853, 2012.

[9] V. Venkatasubramanian, H. Schattler, and J. Zaborszky, "Fast timevarying phasor analysis in the balanced three-phase large electric power system," IEEE Transactions on Automatic Control, vol. 40, no. 11 , pp. 1975-1982, 1995.

[10] A. van der Schaft, L2-Gain and Passivity Techniques in Nonlinear Control. Springer, 2017.

[11] S. Fiaz, D. Zonetti, R. Ortega, J. Scherpen, and A. van der Schaft, "A port-hamiltonian approach to power network modeling and analysis," European Journal of Control, vol. 19, no. 6, pp. 477 - 485, 2013, lagrangian and Hamiltonian Methods for Modelling and Control.

[12] M. Arcak, "Passivity as a design tool for group coordination," IEEE Transactions on Automatic Control, vol. 52, no. 8, pp. 1380-1390, 2007.

[13] M. Perez, R. Ortega, and J. R. Espinoza, "Passivity-based PI control of switched power converters," IEEE Transactions on Control Systems Technology, vol. 12, no. 6, pp. 881-890, Nov 2004.

[14] P. Nahata, R. Soloperto, M. Tucci, A. Martinelli, and G. FerrariTrecate, "A passivity-based approach to voltage stabilization in islanded DC microgrids with ZIP loads,” Tech. Rep., 2017. [Online]. Available: https://infoscience.epfl.ch/record/253266? ?n=en

[15] R. Soloperto, P. Nahata, M. Tucci, and G. Ferrari-Trecate, "A passivitybased approach to voltage stabilization in DC microgrids," in 2018 Annual American Control Conference (ACC), June 2018, pp. 5374 5379

[16] J. Schiffer, D. Zonetti, R. Ortega, A. M. Stanković, T. Sezi, and J. Raisch, "A survey on modeling of microgrids - From fundamental physics to phasors and voltage sources," Automatica, vol. 74, pp. 135150, 2016.

[17] H. K. Khalil, Nonlinear systems (3rd edition). Prentice Hall, 2001.

[18] M. Arcak, C. Meissen, and A. Packard, Networks of dissipative systems: compositional certification of stability, performance, and safety. Springer, 2016.

[19] M. S. Sadabadi, Q. Shafiee, and A. Karimi, "Plug-and-play voltage stabilization in inverter-interfaced microgrids via a robust control strategy," IEEE Transactions on Control Systems Technology, vol. 25, no. 3, pp. 781-791, 2017.

[20] P. Kundur, Power System Stability and Control. McGraw-Hill, 1994.

[21] B. Brogliato, R. Lozano, B. Maschke, and O. Egeland, Dissipative Systems Analysis and Control. London: Springer, 2007.

[22] R. A. Horn and C. R. Johnson, Matrix analysis. Cambridge University Press, 2012. 